\title{
Multiple brain abscesses caused by infection with Candida glabrata: A case report
}

\author{
ZIFENG ZHU ${ }^{1}$, ZHEHAO HUANG ${ }^{1}$, ZHENSHENGNAN LI ${ }^{2}$, XIANGLAN LI $^{3}, \mathrm{CHAO} \mathrm{DU}^{1}$ and YU TIAN ${ }^{1}$ \\ ${ }^{1}$ Department of Neurosurgery, China-Japan Union Hospital of Jilin University, Changchun, Jilin 130033; ${ }^{2}$ Department of \\ Clinical Medicine, Norman Bethune Health Science Center of Jilin University, Changchun, Jilin 130021; ${ }^{3}$ Laboratory of Fungus, \\ Department of Dermatology, China-Japan Union Hospital of Jilin University, Changchun, Jilin 130033, P.R. China
}

Received November 28, 2016; Accepted June 29, 2017

DOI: $10.3892 /$ etm.2018.5692

\begin{abstract}
The present case report described the initial diagnosis of a 25-year old female with a brain abscess consisting of two lesions 0.2 and $2.9 \mathrm{~cm}^{3}$ in volume. The patient was initially treated with antibiotics; however, 2 months following initial treatment, the patient's condition deteriorated and she became vegetative. Following transfer to the China-Japan Union Hospital of Jilin University (Jilin, China) the two lesions had grown in volume to 9.0 and $13.0 \mathrm{~cm}^{3}$, respectively. The results of magnetic resonance spectroscopy and plasma $1-3-\beta$-D-glucan activity suggested a possible fungal infection. Subsequently, a stereotactic biopsy was conducted, fluid was cultured and itraconazole treatment was initiated. Analysis of cultures confirmed a Candida glabrata infection and antifungal treatment was continued. Shortly following surgery, the patient regained consciousness and the ability to eat and speak. A follow-up MRI 8 months following biopsy confirmed disappearance of all lesions and no recurrence. To the best of our knowledge, this is the first English-language report of a brain abscess caused primarily by Candida glabrata.
\end{abstract}

\section{Introduction}

Although $>100$ thousand fungal species are recognized, only a couple of hundred have been suggested to be pathogenic to humans and $10-15 \%$ of pathological fungi produce systemic and/or central nervous system (CNS) mycosis (1).

Species of Candida may cause intracranial abscesses and multiple cases have been described in the literature. The most common identified strain that causes infections is Candida albicans (C. albicans) (2-16); however, C. parapsilosis (17-19),

Correspondence to: Dr Chao Du or Dr Yu Tian, Department of Neurosurgery, China-Japan Union Hospital of Jilin University, 126 Xiantai Street, Changchun, Jilin 130033, P.R. China

E-mail: duchao0987@yahoo.com

E-mail: tianyu@jlu.edu.cn

Key words: Candida glabrata, brain abscess, lesions, stereotactic surgery, magnetic resonance imaging
C. tropicalis (20), C. encephalitis (21) and C. lusitanea (22) infections have also been recorded. In a retrospective analysis of 128 cases of Candida bloodstream infections, the most frequently isolated species were $C$. albicans (64\%), C. glabrata (20\%), C. tropicalis (9\%) and C. parapsilosis (5\%) (23). Intracranial abscesses caused by fungal infections may present with various clinical syndromes, including basal meningitis, space occupying lesions, stroke syndromes, hydrocephalus and spinal infections (1). Compared to viral, bacterial or parasitic CNS disorders, symptomatic CNS fungal infection carries higher risks of morbidities and mortality (1). Different from other Candida species, C. glabrata is found as blastoconidia as a commensal and a pathogen, and infections are difficult to treat and typically resistant to various azole antifungal agents, particularly fluconazole (24-26). Consequently, C. glabrata infections have a high mortality rate in compromised, at-risk hospitalized patients (27). Although $C$. glabrata has been identified as causing infections of the bloodstream, to the best of our knowledge, the current report is the first to describe an intracranial abscess caused by $C$. glabrata.

Itraconazole has in vitro activity against many of the non-albicans Candida species, specifically C. glabrata; furthermore, an improvied response rate to itraconazole has been indicated compared with fluconazole (27). The present case study reports the successfully treated cerebral abscess case due to $C$. glabrata specifiying the surgical approach and itraconazole treatment. Furthermore, the present study reviewed the literature regarding this infection.

\section{Case report}

Approval regarding the use of human data for experimental and clinical studies was obtained from the Ethics Committee of the China-Japan Union Hospital of Jilin University (Jilin, China) and written informed consent was obtained from the patient's family. The principles expressed in the Declaration of Helsinki were adhered to in order to maintain the integrity of the data.

The current study describes the case of a 25-year-old female patient, weighing $40 \mathrm{~kg}$ who presented with fever $\left(38.7-39.0^{\circ} \mathrm{C}\right)$, phlegmy cough and numbness in the right hand. Within $24 \mathrm{~h}$ onset of these symptoms, the patient was unable to speak or move the upper and lower limbs on the right-hand side. 
The patient had undergone an anal polypectomy on November 10, 2014 at the Dehui Municipal Hospital (Jilin, China) to remove a flat, soft, polyp $5 \mathrm{~cm}$ in size that had been present outside the anus for $>7$ years. Pathology indicated that it was a benign papilloma. Relevant medical history included frequent colds and fevers ranging between $38-39^{\circ} \mathrm{C}$, psoriasis involving the scalp, abdomen and all limbs from the age of 8 and a 12-year history of smoking ( 20 cigarettes/day).

On December 29, 2014, the patient was admitted to the Neurology Clinic at the First Hospital of Jilin University with lethargy, uncooperativeness during physical examination, motor aphasia, left central facial paralysis, strong nuchal rigidity, level 1right limb muscle strength, increased muscle tone, hyperreflexia and level 5 left limb muscle strength. The patient exhibited a positive bilateral Kernig sign, bilateral positive Babinski signs and bilateral positive Chaddock sign, which were indicative of meningeal irritation and pyramidal sign positive, is the clinical features of meningitis and pyramidal tract damage. However, peripheral blood and cerebrospinal fluid cultures tested negative for bacteria and fungi. An ultrasonograpic examination indicated no perianal abscess. A computerized tomography (CT) scan of the lungs identified minor inflammatory changes in the right lung in all lobes and the lingula and lower lobe of the left lung. A CT scan of the head revealed multiple low-density patches of different sizes in the right frontal lobe, left corona radiate and left half-oval center. The CT value was 16-26 HU and the midline had not shifted. Minor inflammation was present on the bilateral maxillary sinuses and mastoid inflammation was evident in the middle ears. The patient was hospitalized on December 29, 2014 and began treatment with $125 \mathrm{ml}$ mannitol three times a day via intravenous injection.

On December 31, 2014, the patient underwent a magnetic resonance imaging (MRI) scan of the head at the First Hospital of Jilin University. The results revealed multiple lesions and abnormal enhancements in the intracranial bilateral frontal and temporal lobes. Furthermore, the midline was shifted slightly to the right. Lesions were detected in the right frontal lobe (size, 8x8x8 mm; volume, $0.2 \mathrm{~cm}^{3}$; Fig. 1A-C) and left lateral temporal lobe (size, 19x19x20 mm; volume, $2.9 \mathrm{~cm}^{3}$; Fig. 1D-F). Cerebrospinal fluid (CSF) examination (3 tubes were collected, $2 \mathrm{ml}$ per tube) identified colorless CSF with a pressure of $110 \mathrm{mmH}_{2} \mathrm{O}$ (normal range, 80-180 $\mathrm{mmH}_{2} \mathrm{O}$ ). The protein, glucose and chlorine levels in the CSF of the patient were $0.46 \mathrm{~g} / \mathrm{l}$ (normal range, 0.12-0.60 g/l), $3.49 \mathrm{mmol} / \mathrm{l}$ (normal range, 2.2-3.9 $\mathrm{mmol} / \mathrm{l}$ ) and $129.3 \mathrm{mmol} / \mathrm{l}$ (normal range, $120.0-132.0 \mathrm{mmol} / \mathrm{l}$ ), respectively. The patient exhibited a weakly positive Pandy's reaction. The patient's white blood cell (WBC) and erythrocyte count in the CSF was 599x10 1 (normal range, $0-8 \times 10^{6} /$ ) and $200 \times 10^{6} / 1$ (normal range, $0 \times 10^{6} / 1$ ), respectively and multinucleate cell and monocyte levels were 9 and $91 \%$, respectively. The level of immunoglobulin (Ig) G in the CSF was $15.90 \mathrm{mg} / \mathrm{l}$ (normal range, $0-34.0 \mathrm{mg} / \mathrm{l}$ ) and the level of high-sensitivity C-reactive protein (CRP) was $7.51 \mathrm{mg} / \mathrm{l}$ (normal range, 0.0-5.0 mg/l). Hematological evaluation was performed in the 4-ml blood sample extracted from the patient. The sample was centrifuged at $1,342 \times \mathrm{g}$ at $4^{\circ} \mathrm{C}$ for $10 \mathrm{~min}$ before a WBC count of $11.12 \times 10^{9} / 1$ (normal range, $4.0-10.0 \times 10^{9} / 1$ ), neutrophil percentage of $79 \%$ (normal range, $50-70 \%$ ) and absolute neutrophil count of $8.73 \times 10^{9} / 1$ (normal range, $\left.2.0-7.0 \times 10^{9} / 1\right)$ were determined. The results of tests for syphilis and human immunodeficiency virus (HIV) were negative. The patient was diagnosed with a brain abscess, purulent meningitis and bilateral pneumonia. Vancomycin ( $1.0 \mathrm{~g}$, twice a day) and meropenem (1.0 g, 6 times a day) were administered via intravenous injection. However, 11 days after this treatment was initiated, symptoms had worsened and the patient was unable to eat.

On January 19, 2015, the patient was transferred back to Dehui Municipal Hospital. The patient continued to receive treatment with vancomycin but not meropenem until a rash developed 10 days later and antibiotic therapy was stopped.

On January 29, 2015, the patient was hospitalized and examined at the neurosurgery clinic of the China-Japanese Union Hospital of Jilin University. Physical examination demonstrated that the patient was in a vegetative state. The patient was able to open their eyes but was unable to move them and unable to speak. The patient exhibited high tension in the lower limb muscles and stiff muscles. An MRI scan (non-contrast and contrast-enhanced) conducted $48 \mathrm{~h}$ after admittance to the China-Japanese Union Hospital revealed sheet-like and patch-like lesions on T1 and mixed isointense areas on T2 in the bilateral frontal lobe, parietal lobe, bilateral corona radiata area, semi-oval center and right occipital lobe. The internal signals were uneven, exhibiting a number of round-shaped hypointense lesions on T1 and hyperintense lesions on T2. The left frontal lobe exhibited an abnormal signal consisting of linear-shaped lesions that may have been caused by bleeding. The enhanced scan suggested that the lesions were diffuse and consisted of small patchy areas of ring-like enhancement surrounding them. The abscess in the left frontal cavity had increased to a volume of $13.0 \mathrm{~cm}^{3}$ (size, 26x26x35 mm; Fig. 2A, C, E and F). The abscess in the left semi-oval center cavity had increased to a volume of $9.0 \mathrm{~cm}^{3}$ (size, 25x22x30 mm; Fig. 2B, D-F). Magnetic resonance spectroscopy (MRS) was conducted and the solid entity on the cavity wall was scanned. The results identified an $\mathrm{N}$-acetyl aspartate peak, a significant decrease in the creatine peak, an elevated choline peak, dual lactate (Lac) peaks (1.1-1.5 ppm) and a visible inositol peak (3.56-4.06 ppm) (data not shown). Following previous treatment with vancomycin and meropenem for 1 month, which had led to the deterioration of the patient's condition, medical therapy was changed to $1.0 \mathrm{~g}$ vancomycin in combination with $2.0 \mathrm{~g}$ ceftriaxone twice a day via intravenous injection.

A total of 2 days after admission, the patient's peripheral blood was examined for Ig levels ( $5 \mu \mathrm{l}$ blood; centrifuged at $986 \mathrm{x} \mathrm{g}$, at $4^{\circ} \mathrm{C}$ for $\left.10 \mathrm{~min}\right)$ and complement proteins $(3 \mu \mathrm{l}$ blood; centrifuged at $986 \times \mathrm{g}$, at $4^{\circ} \mathrm{C}$ for $10 \mathrm{~min}$ ). The levels of $\mathrm{IgG}, \mathrm{IgM}$ and $\mathrm{IgA}$ were $4.65 \mathrm{~g} / \mathrm{l}$ (normal range, 8-16 g/l), $0.06 \mathrm{~g} / \mathrm{l}$ (normal range, $0.5-2.2 \mathrm{~g} / \mathrm{l}$ ) and $0.525 \mathrm{~g} / \mathrm{l}$ (normal range, 0.7-3.3 g/l), respectively. The levels of complement C3 and complement $\mathrm{C} 4$ were $0.788 \mathrm{~g} / \mathrm{l}$ (normal range, $0.9-1.5 \mathrm{~g} / \mathrm{l}$ ) and $0.236 \mathrm{~g} / \mathrm{l}$ (normal range, 0.2-0.4 g/l), respectively. Plasma $1-3-\beta$-D-glucan activity (4 ml blood; centrifuged at $986 \mathrm{x} \mathrm{g}$, at $4^{\circ} \mathrm{C}$ for $1 \mathrm{~min}$ ), a fungal surrogate marker, was elevated at $225.50 \mathrm{pg} / \mathrm{ml}$ (normal range, $100.50-151.50 \mathrm{pg} / \mathrm{ml}$ ). The patient remained negative for HIV. Taken together, these results suggested the possibility of a fungal infection; therefore, the patient was prepared for stereotactic surgery to target the intracranial lesions. 
A

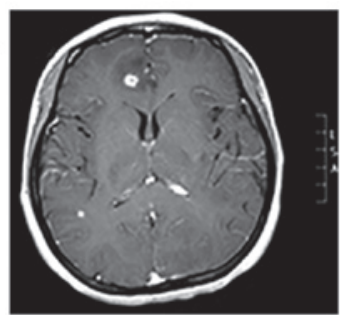

D

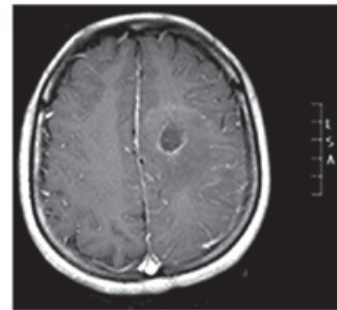

B

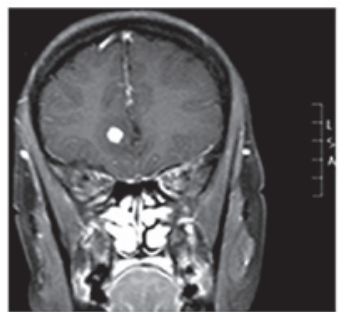

E

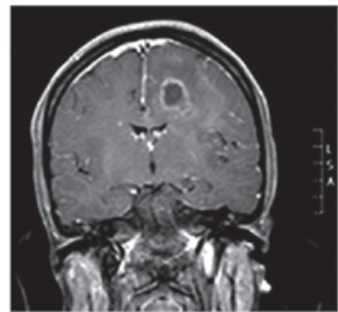

C

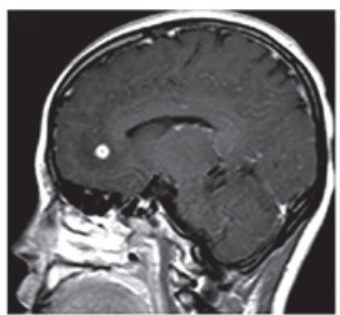

$\mathbf{F}$

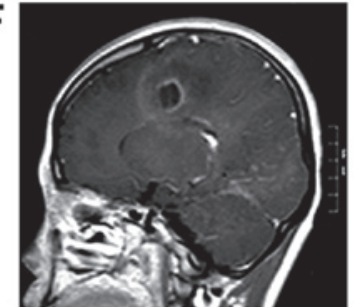

Figure 1. Initial magnetic resonance imaging scan indicating two brain abscess lesions. (A-C) Right frontal lobe lesions (size: $8 \mathrm{x} 8 \mathrm{x} 8 \mathrm{~mm}$, volume $0.2 \mathrm{~cm}{ }^{3}$ ). (D-F) Left temporal lobe lesions (size: 19x19x20 mm, volume $2.9 \mathrm{~cm}^{3}$ ). (A and D) Axial contrast-enhanced T1WI. (B and E) Coronal contrast-enhanced T1WI. (C and F) Sagittal contrast-enhanced T1WI. T1W1, T1 weighted image.

\section{A}

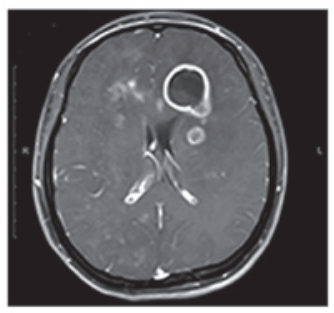

B

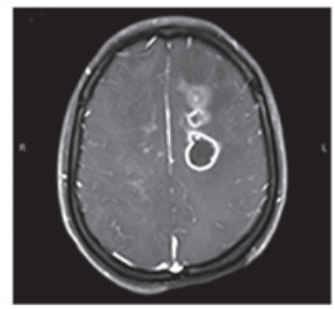

D

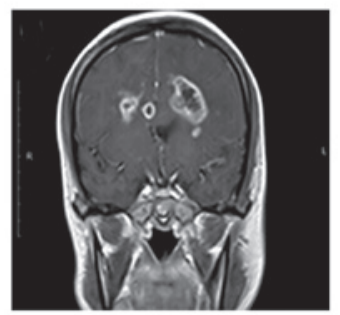

E

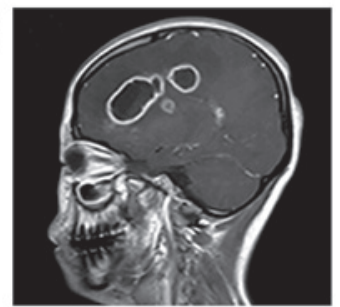

C

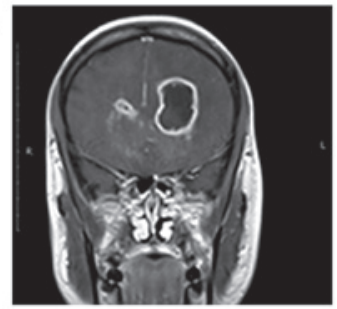

$\mathbf{F}$

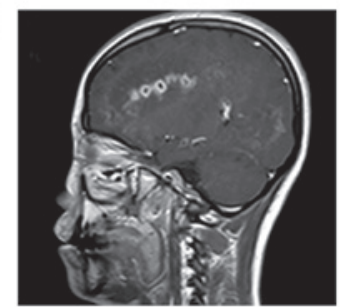

Figure 2. Magnetic resonance imaging scans following 1 month treatment indicating an increase in size of brain abscess lesions. The cavity of the abscess in the left frontal had a size of 26x26x35 mm and its volume increased to $13.0 \mathrm{~cm}^{3}$. The cavity of the abscess in the left semi-oval center was $25 \times 22 \times 30 \mathrm{~mm}$ and its volume increased to $9.0 \mathrm{~cm}^{3}$. (A) Axial contrast-enhanced image of the abscess in the left frontal lobe. (B) Axial contrast-enhanced image of the abscess in the left semi-oval center. (C) Coronal contrast-enhanced image of the abscess in the left frontal lobe. (D) Coronal contrast-enhanced image of the abscess in the left semi-oval center ( $\mathrm{E}$ and F) sagittal contrast-enhanced image of the abscess in the left frontal lobe and left semi-oval center.

A total of 7 days after admission, the patient underwent a stereotactic biopsy of aspirate fluid from the intracranial cyst using Komai's stereotactic instrument (Fig. 3A and B). The CT-Stereotactic Frame used in the current study was manufactured by Mizuho Medical Co., Ltd. (Tokyo, Japan). The left frontal lobe lesion was initially targeted (Fig. 3C). During the attempt to puncture the target lesion, the needle encountered significant resistance prior to reaching the center. The texture was flexible and resistant and led to the hypothesis that the resistance encountered was the lesion wall. Following successful insertion of the needle into the abscess cavity, a syringe was used to aspirate $5.0 \mathrm{ml}$ pale green fluid and $3.0 \mathrm{ml}$ off-white, toothpaste-like cystic tissue. A silicone tube was then placed to for further drainage. The target was adjusted and subsequently, the lesion in the left semi-oval center was punctured (Fig. 3D). Using a spiral tissue biopsy needle, the lesion wall tissue, which looked like an off-white, cheese-like substance to the naked eye, was partially removed. A total of $3.0 \mathrm{ml}$ yellow-white, granular, millet-like content was also removed. None of the aspirated materials exhibited a noxious odor.

Following this procedure, it was considered that the patient may have a fungal infection. Following surgery, antibiotic treatment was attenuated and itraconazole solution $(200 \mathrm{mg} /$ day; Janssen Pharmaceutica N.V., Beeerse, Belgium) antifungal treatment was administered twice daily via a nasogastric tube.

A CT scan of the head was conducted 1 day following the stereotactic surgery. This confirmed that the abscess in the left frontal cavity was notably smaller and a shadow of gas was present in the brain parenchyma (Fig. 3E). The drainage tube was positioned at the center of the abscess. The abscess in the left semi-oval center was also noticeably smaller (Fig. 3F). A 

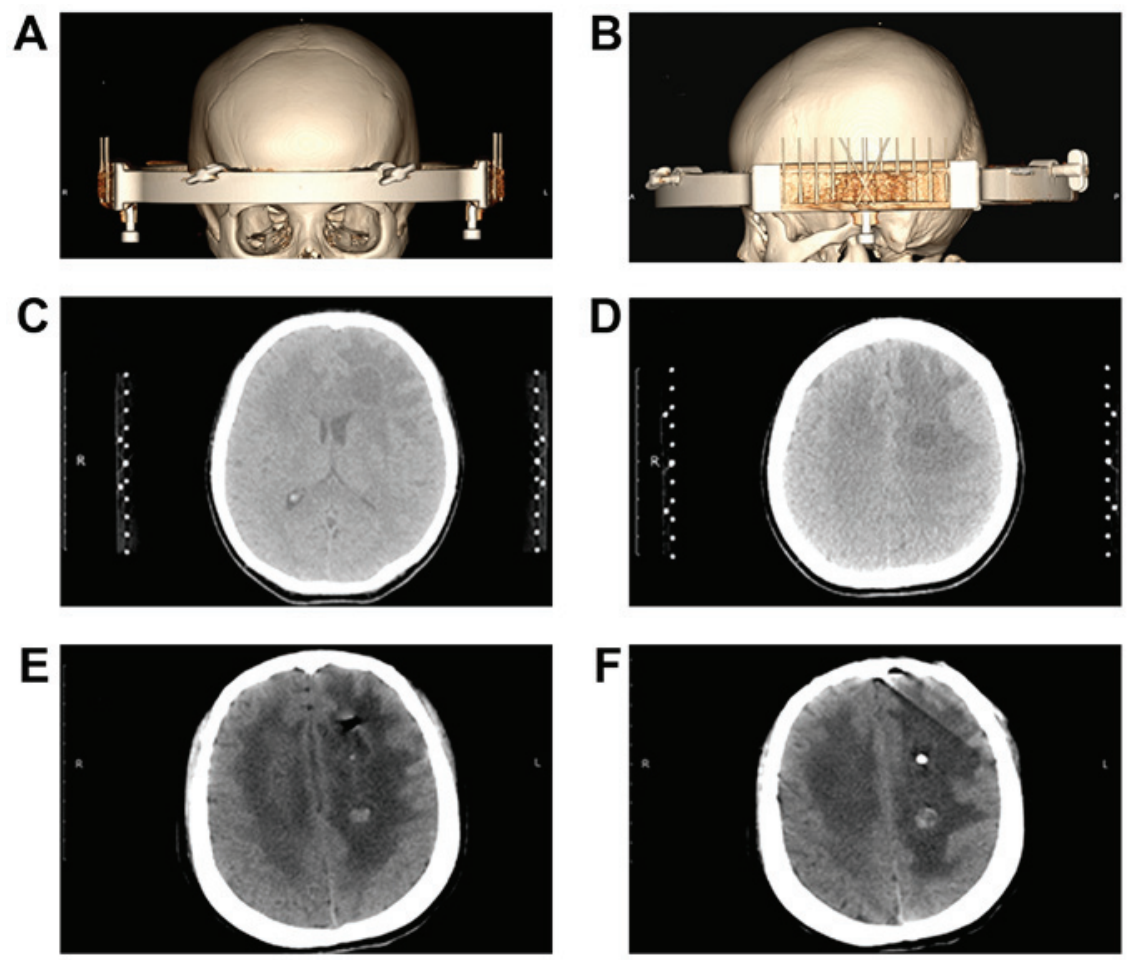

Figure 3. 3D CT images of stereotactic biopsy and cyst fluid aspiration surgery. (A) Stereotactic surgery localization and an axial image of 3D CT reconstruction. (B) Stereotactic surgery localization and sagittal image of 3D CT reconstruction. (C) Stereotactic surgery targeting the left frontal lesion selection. (D) Stereotactic surgery targeting the left semi-oval center lesion. (E) CT scan of the head conducted 1 day following stereotactic biopsy indicating a decrease in the size of the abscess in the left frontal cavity and a shadow of gas present in the brain parenchyma. (F) CT scan of the head conducted 1 day following stereotactic biopsy indicating the drainage tube positioned in the center of the abscess. The abscess in the left semi-oval center was notably smaller. A round and high-density shadow was present in the cavity. CT, computer topography; 3D, three-dimensional.
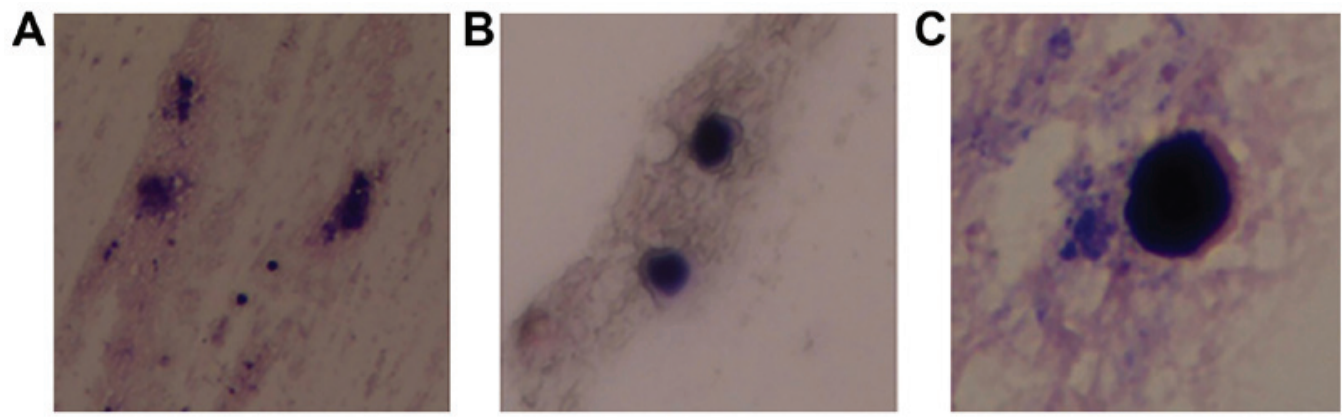

Figure 4. Cystic fluid cultured in CHROMagar Candida chromogenic medium indicated the presence of Candida glabrata. Magnifications of (A) x100, (B) $\mathrm{x} 400$ and (C) $\mathrm{x} 1,000$.

round, high-density shadow was present in the cavity, potentially as a result of minimal bleeding following the biopsy on the wall of the abscess cavity (Fig. 3F).

Tissue and aspirated fluids were sent to the pathology laboratory for analysis. Tissue from the cavity wall indicated local brain tissue gliosis and the presence of scattered inflammatory cells. Cystic fluid was cultured in CHROMagar Candida chromogenic medium (Chromagar; Paris, France). Cystic fluid was streaked onto sterile Petri dishes which contain $10 \mathrm{ml}$ CHROMagar Candida chromogenic medium, and incubated in aerobic conditions at $35^{\circ} \mathrm{C}$.) and purple colony growth was present following 7 days in culture. Following 2 weeks of culture, the medium exhibited a scattered, gram-staining, oval yeast that was identified as $C$. glabrata (Fig. 4). The steps of gram-staining were as follows: The colony was smeared on the slide, stained with crystal violet for $1 \mathrm{~min}$, rinsed gently with water, saturated with iodine for $1 \mathrm{~min}$, rinsed with water again and decolorized with $75 \%$ alcohol for 20 sec. Subsequently, the slide was rinsed with water, counterstained with safranin for $1 \mathrm{~min}$ and rinsed with water again. The slide was observed under a light microscope. The cultured $C$. glabrata did not present any blastospores or mycelium, suggesting that the strain was in a low-activity state.

A total of 4 days following surgery, the patient regained consciousness and regained the ability to speak. Right limb muscle strength improved from level 2 to level 5. Levels of plasma 1-3- $\beta$-D-glucan decreased to $88.20 \mathrm{pg} / \mathrm{ml}$ by February 14, 2015. The patient was discharged 10 days following surgery and began eating the following day. After a further 9 days, the patient was able to move the limbs on their right side. On March 5, 2015, the 

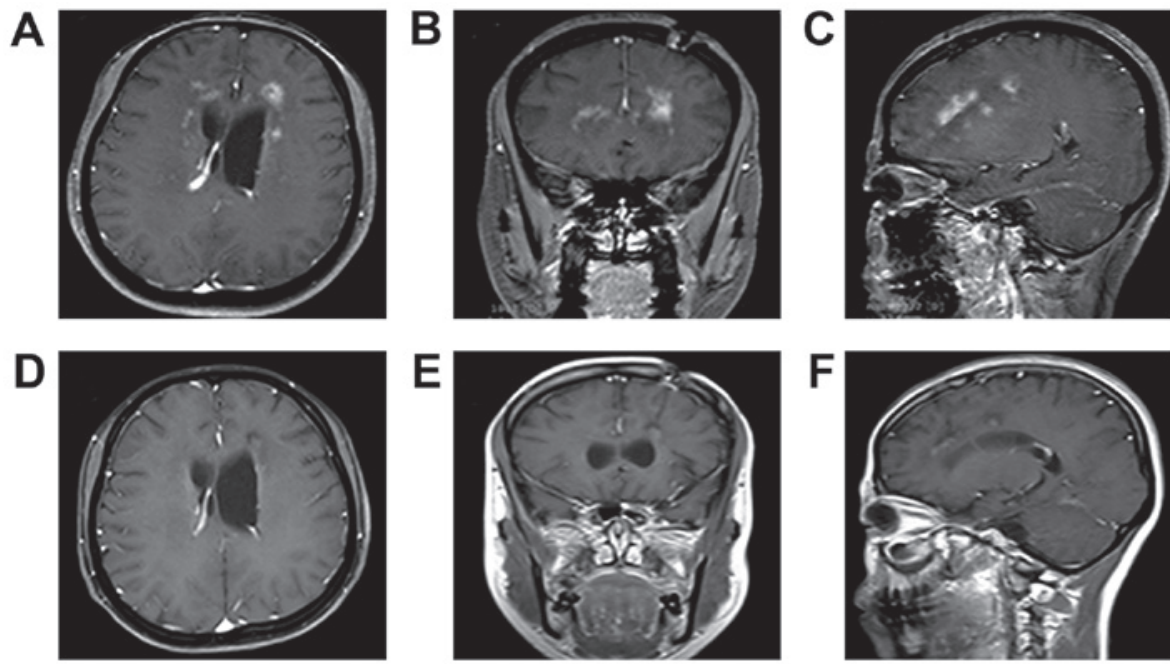

Figure 5. Follow-up magnetic resonance imaging images indicated no recurrence of Candida glabrata infection. (A-C) The lesion in the left frontal lobe decreased to a size of $12 \times 11 \times 10 \mathrm{~mm}$ and a volume of $0.7 \mathrm{~cm}^{3}$ and the remaining original lesions exhibited no enhancement 2.5 months following stereotactic operation. (D-F) All lesions had disappeared 6 months following stereotactic operation. (A and D) Axial contrast-enhanced T1WI. (B and E) Coronal contrast-enhanced T1WI. (C and F) Sagittal contrast-enhanced T1WI; T1W1, T1 weighted image.

patient regained the ability to speak in complete sentences and was able to walk again on Apri 12,2015.

A follow-up examination on April 20, 2015 included an MRI scan. A mild ring-like enhancement indicated the presence of a $0.7 \mathrm{~cm}^{3}(12 \times 11 \times 10 \mathrm{~mm})$ single lesion in the left frontal lobe. There was no enhancement shadow in place of the original lesions (Fig. 5A-C). The patient underwent 20-day, 1-month, 2-month and 2.5-month follow-up examinations to confirm full recovery of speech and mobility. During these follow-ups the patient reported a change in their eating habits, with an increased preference for meat instead of vegetables and also gained weight. An additional follow-up MRI scan conducted 8 months after surgery, which indicated the disappearance of all lesions (Fig. 5D-F), confirming treatment success. Up to now (24-month follow-up), there was no recurrence of $C$. glabrata infection.

\section{Discussion}

Candida infections occur most often in patients with severe immune function defects (28), such as HIV infections (29). However, some patients develop brain abscesses following bone marrow transplantation (30). Although the patient in the current report did not have a history of compromised immune function, examination of peripheral blood for Ig levels and complement tests revealed lower than normal levels of $\mathrm{IgG}$, IgM and complement $\mathrm{C} 3$, indicating an immune system abnormality. Cerebral abscesses secondary to Candida infection have been reported in previous studies $(3,8,16)$; however, in the current study, it is considered that the patient's brain abscesses were primarily caused by $C$. glabrata.

The patient was initially diagnosed with a brain abscess by MRI and CSF examinations. It was confirmed following hospitalization that infection was not present in any other organs and there was no history of antibiotic use within 6 months of hospitalization. Following treatment with antibiotics, the patient's condition deteriorated. The patient lost the ability to eat and talk, and subsequently fell into a vegetative state. Additional tests were conducted, including tests for CRP and the fungal surrogate marker, plasma $1-3-\beta-D$-glucan. The results of these tests suggested the possibility of a fungal infection. An MRI scan of the head identified that the abscesses had increased in size; therefore, the patient was prepared for stereotactic surgery to target the intracranial lesions. A section of the cystic wall was removed and aspirated fluid and tissue were removed from intracranial lesions with no complications. The patient underwent treatment with itraconazole and 1 day following surgery, the lesions were noticeably smaller. The patient's plasma 1-3- $\beta$-D-glucan value decreased to within normal limits following treatment. The patient regained consciousness, right limb muscle strength and was able to speak 4 days following stereotactic surgery. Tissues and fluid were cultured for 2 weeks and $C$. glabrata was identified.

Although there have been numerous advances in diagnostic procedures and therapeutic strategies, patients with fungal infections of the brain typically have a poor prognosis (2). Factors associated with the poor prognosis of patients with Candida meningitis include: At $>2$ weeks prior to diagnosis of Candida meningitis, onset of intracranial hypertension, appearance of focal neurologic deficits and decreased glucose levels in the CSF $(<35 \mathrm{mg} / \mathrm{dl})$ was indicated (31). Successful management of patients requires practitioners to have a high degree of suspicion of a cerebral fungal infection, even as the severity of symptoms varies $(12,32,33)$. Diagnosis is often difficult, as the results of neuroradiological investigations, including CT scans, MRS, radiography and laboratory assessments are often inconclusive $(11,12)$. There have been several cases in which patients have experienced a loss of consciousness, fever, focal neurological deficits, headaches and seizures; however, examinations of the CSF, peripheral blood, urine and/or CT scans have failed to uncover the underlying cause(s) of their neurological issues $(11,12,34)$. In addition, it can be difficult to differentiate between patients with cerebral aspergillosis and cerebral candidiasis since the two infections manifest with similar clinicopathological features (32). However, infection of the sino-nasal tract is 
more common in patients with cerebral aspergillosis, whereas infections of the gastrointestinal tract are more common in patients with cerebral candidiasis (32). In the current report, it was hypothesized that the initial site of infection was the perineum and occurred following the patient's anal polypectomy.

A surgical approach combined with medical therapy is the most successful method of treating suspected fungal central nervous system (CNS) infections (2). Patients with intracranial fungal masses may be treated with stereotactic biopsy and/or partial or radical surgical excision of the mass(es), coupled with intravenously or intrathecally administered antifungal therapy $(28,35)$. The most widely used medications for patients with CNS mycoses include liposomal amphotericin B, 5-fluorocytosine, fluconazole and itraconazole $(28,31)$. In the current study, a stereotactic biopsy was performed on the patient. Tissue samples and aspirated fluids were sent to a pathology laboratory and after 2 weeks, C. glabrata was identified in culture. MRS was conducted during diagnosis of the patient. Although the patient exhibited a Lac peak, which is indicative of anaerobic metabolism in the lesion and suggestive of inflammatory processes, the results of MRS were inconclusive. Therefore, the use of MRS does not replace the requirement for tissue samples when diagnosing a brain abscess.

The outcome for the majority of patients with CNS fungal infections is poor. Mortality rates are $\sim 50 \%$ which is attributed to delayed diagnosis and onset of treatment, the presence of serious underlying diseases or conditions, including HIV, transplant recipients and hematological malignancies, and the existence of multi-drug-resistant fungal organisms $(5,28)$. In a study investigating patients undergoing bone marrow transplantation with a risk of mortality unrelated to the etiology of the brain abscess or choice of therapeutic regimen, the mortality rate was $97 \%$ (30). In cases where patients survive fungal CNS infections, they often face long-term neurological sequelae (28). Infection with Candida species has become the most prevalent cerebral mycosis detected at autopsy $(2,11,12,31)$, indicating ineffective and/or inadequate eradication (19).

In the current study, a final diagnosis of $C$. glabrata cerebral mycosis was made following pathological examination of tissue and fluid samples obtained during stereotactic biopsy. Treatment with antifungal therapy led to recovery and the patient experienced no further neurological sequelae. Assessment of the patient's immunological status during the course of illness revealed lower than normal levels of $\mathrm{IgG}, \mathrm{IgM}$ and complement $\mathrm{C} 3$, indicating the presence of an underlying immune system abnormality. Information gleaned from previous studies and the current case report have demonstrated that timing, neuroradiological investigations and typical clinical laboratory assessments, such as examination of CSF, do not lead to the diagnosis of cerebral mycosis.

In conclusion, all brain abscesses, including small abscesses should be biopsied as early as possible so that objective evidence of the underlying cause of the disease, particularly regarding the type of mycosis, maybe identified as early as possible following onset. To the best of our knowledge, the current case report is the first to be published in English describing a brain abscess caused primarily by $C$. glabrata.

\section{Acknowledgements}

The present case report was supported by the Science and Technology Department of Jilin Province, China (grant nos. 20110472 and 20150204072 SF).

\section{References}

1. Raman Sharma R: Fungal infections of the nervous system: Current perspective and controversies in management. Int J Surg 8: 591-601, 2010.

2. Neves N, Santos L, Reis C and Sarmento A: Candida albicans brain abscesses in an injection drug user patient: A case report. BMC Res Notes 7: 837, 2014

3. Wang GH, Dai CL, Liu YF and Li YM: Cerebral and renal abscess and retino-choroiditis secondary to Candida albicans in preterm infants: Eight case retrospective study. Clin Exp Obstet Gynecol 40: 519-523, 2013.

4. Ancalle IM, Rivera JA, Garcia I, Garcia L and Valcárcel M: Candida albicans meningitis and brain abscesses in a neonate: A case report. Bol Asoc Med P R 102: 45-48, 2010.

5. Njambi S, Huttova M, Kovac M, Freybergh PF, Bauer F and Muli JM: Fungal neuroinfections: Rare disease but unacceptably high mortality. Neuro Endocrinol Lett 28 (Suppl 2): S25-S26, 2007.

6. Prabhu RM and Orenstein R: Failure of caspofungin to treat brain abscesses secondary to Candida albicans prosthetic valve endocarditis. Clin Infect Dis 39: 1253-1254, 2004.

7. Marcinkowski M, Bauer K, Stoltenburg-Didinger $G$ and Versmold H: Fungal brain abscesses in neonates: Sonographic appearances and corresponding histopathologic findings. J Clin Ultrasound 29: 417-421, 2001.

8. Gözdaşoĝlu S, Ertem M, Büyükkeçeci Z, Yavuzdemir S, Bengisun S, Ozenci H, Taçyildiz N, Unal E, Yavuz G, Deda G and Aysev D: Fungal colonization and infection in children with acute leukemia and lymphoma during induction therapy. Med Pediatr Oncol 32: 344-348, 1999.

9. Burgert SJ, Classen DC, Burke JP and Blatter DD: Candidal brain abscess associated with vascular invasion: A devastating complication of vascular catheter-related candidemia. Clin Infect Dis 21: 202-205, 1995.

10. Kamitsuka MD, Nugent NA, Conrad PD and Swanson TN: Candida albicans brain abscesses in a premature infant treated with amphotericin B, flucytosine and fluconazole. Pediatr Infect Dis J 14: 329-331, 1995.

11. Radhakrishnan VV, Saraswathy A, Rout D and Mohan PK: Disseminated intra-cerebral microabscesses: A clinico-pathologic study. Indian J Pathol Microbiol 37: 171-178, 1994.

12. Pendlebury WW, Perl DP and Munoz DG: Multiple microabscesses in the central nervous system: A clinicopathologic study. J Neuropathol Exp Neurol 48: 290-300, 1989.

13. Wiethölter H, Thron A, Scholz E and Dichgans J: Systemic Candida albicans infection with cerebral abscess and granulomas. Clin Neuropathol 3: 37-41, 1984

14. Thron A and Wiethölter H: Cerebral candidiasis: CT studies in a case of brain abscess and granuloma due to Candida albicans. Neuroradiology 23: 223-225, 1982.

15. Holyst J, Majewski A and Tyszkiewicz S: Massive cerebellar abscess due to Candida albicans. Neurochirurgia (Stuttg) 19: 126-129, 1976.

16. Black JT: Cerebral candidiasis: Case report of brain abscess secondary to Candida albicans, and review of literature. J Neurol Neurosurg Psychiatry 33: 864-870, 1970.

17. Bagheri F, Cervellione KL, Maruf M, Marino W and Santucci T Jr: Candida parapsilosis meningitis associated with shunt infection in an adult male. Clin Neurol Neurosurg 112: 248-251, 2010.

18. Andres RH, Guzman R, Weis J, Schroth G and Barth A: Granuloma formation and occlusion of an unruptured aneurysm after wrapping. Acta Neurochir (Wien) 149: 953-958, 2007.

19. Lipton SA, Hickey WF, Morris JH and Loscalzo J: Candidal infection in the central nervous system. Am J Med 76: 101-108, 1984.

20. Yoganathan S, Chakrabarty B, Gulati S, Kumar A, Kumar A, Singh $\mathrm{M}$ and Xess I: Candida tropicalis brain abscess in a neonate: An emerging nosocomial menace. Ann Indian Acad Neurol 17: 448-450, 2014. 
21. Maschke M, Dietrich U, Prumbaum M, Kastrup O, Turowski B, Schaefer UW and Diener HC: Opportunistic CNS infection after bone marrow transplantation. Bone Marrow Transplant 23: 1167-1176, 1999.

22. Antunes NL, Hariharan S and DeAngelis LM: Brain abscesses in children with cancer. Med Pediatr Oncol 31: 19-21, 1998.

23. Schelenz S and Gransden WR: Candidaemia in a London teaching hospital: Analysis of 128 cases over a 7-year period. Mycoses 46: 390-396, 2003.

24. Hitchcock CA, Pye GW, Troke PF, Johnson EM and Warnock DW: Fluconazole resistance in Candida glabrata. Antimicrob Agents Chemother 37: 1962-1965, 1993.

25. Komshian SV, Uwaydah AK, Sobel JD and Crane LR: Fungemia caused by Candida species and Torulopsis glabrata in the hospitalized patient: Frequency, characteristics, and evaluation of factors influencing outcome. Rev Infect Dis 11: 379-390, 1989.

26. Willocks L, Leen CL, Brettle RP, Urquhart D, Russell TB and Milne LJ: Fluconazole resistance in AIDS patients. J Antimicrob Chemother 28: 937-939, 1991.

27. Fidel PL Jr, Vazquez JA and Sobel JD: Candida glabrata: Review of epidemiology, pathogenesis, and clinical disease with comparison to C. albicans. Clin Microbiol Rev 12: 80-96, 1999.

28. Selby R, Ramirez CB, Singh R, Kleopoulos I, Kusne S, Starzl TE and Fung J: Brain abscess in solid organ transplant recipients receiving cyclosporine-based immunosuppression. Arch Surg 132: 304-310, 1997.

29. Pham LV, Quang AT, Ton Nu PA, Duc TT and Thi HN: Cladophialophora bantiana and Candida albicans mixed infection in cerebral abscess of an HIV-negative patient. J Infect Dev Ctries 2: 245-248, 2008.
30. Hagensee ME, Bauwens JE, Kjos B and Bowden RA: Brain abscess following marrow transplantation: Experience at the Fred Hutchinson cancer research center, 1984-1992. Clin Infect Dis 19: 402-408, 1994.

31. Sanchez-Portocarrero J,Pérez-Cecilia E, Corral O, Romero-Vivas J and Picazo JJ: The central nervous system and infection by Candida species. Diagn Microbiol Infect Dis 37: 169-179, 2000.

32. Larbcharoensub N, Wongwichai S, Chongtrakool P, Boongird A, Noinang A, Watcharananan SP, Tunlayadechanont S, Witoonpanich R and Phudhichareonrat S: Cerebral aspergillosis and cerebral candidiasis; a retrospective analysis of clinicopathologic features in Ramathibodi Hospital. J Med Assoc Thai 93: 1443-1450, 2010

33. Fennelly AM, Slenker AK, Murphy LC, Moussouttas M and DeSimone JA: Candida cerebral abscesses: A case report and review of the literature. Med Mycol 51: 779-784, 2013.

34. Kaji M, Shoji H and Oizumi K: Intractable meningitis and intracranial abscess following sinusitis due to Candida species. Kurume Med J 45: 279-281, 1998.

35. Yampolsky C, Corti M and Negroni R: Fungal cerebral abscess in a diabetic patient successfully treated with surgery followed by prolonged antifungal therapy. Rev Iberoam Micol 27: 6-9, 2010.

This work is licensed under a Creative Commons International (CC BY-NC-ND 4.0) License. 